Document downloaded from:

http://hdl.handle.net/10251/147538

This paper must be cited as:

Bernardes, NC.; Bonilla, A.; Peris Manguillot, A.; Wu, X. (2018). Distributional chaos for operators on Banach spaces. Journal of Mathematical Analysis and Applications. 459(2):797-821. https://doi.org/10.1016/j.jmaa.2017.11.005

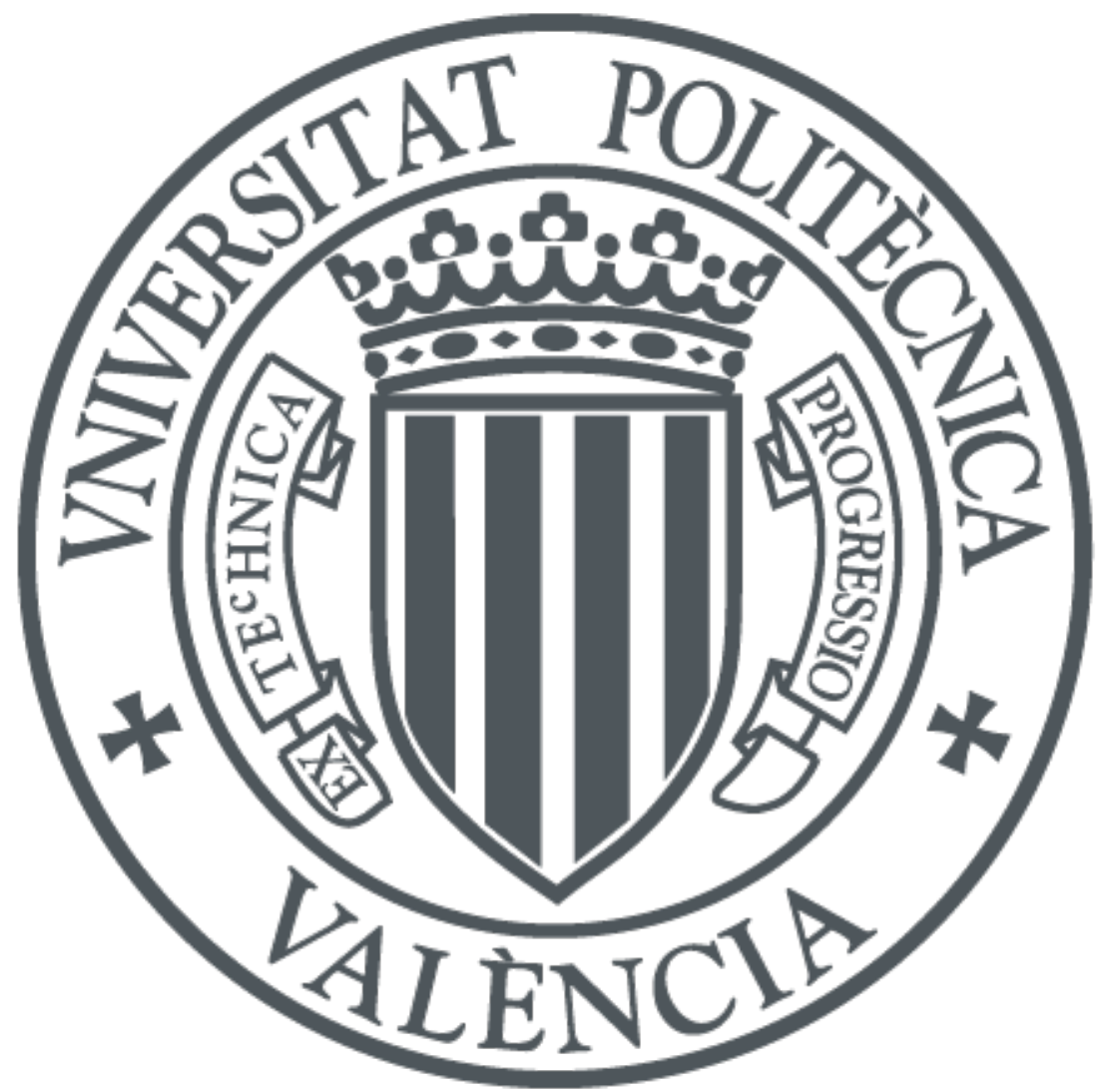

The final publication is available at

https://doi.org/10.1016/j.jmaa.2017.11.005

Copyright Elsevier

Additional Information 


\title{
Distributional chaos for operators on Banach spaces
}

\author{
N. C. Bernardes Jr., A. Bonilla, A. Peris, X. Wu
}

\begin{abstract}
Four notions of distributional chaos, namely DC1, DC2, DC $2 \frac{1}{2}$ and DC3, are studied within the framework of operators on Banach spaces. It is known that, for general dynamical systems, $\mathrm{DC} 1 \subset \mathrm{DC} 2 \subset \mathrm{DC} 2 \frac{1}{2} \subset \mathrm{DC} 3$. We show that $\mathrm{DC} 1$ and $\mathrm{DC} 2$ coincide in our context, which answers a natural question. In contrast, there exist DC2 $\frac{1}{2}$ operators which are not DC2. Under the condition that there exists a dense set $X_{0} \subset X$ such that $T^{n} x \rightarrow 0$ for any $x \in X_{0}$, DC3 operators are shown to be DC1. Moreover, we prove that any upper-frequently hypercyclic operator is DC2 $\frac{1}{2}$. Finally, several examples are provided to distinguish between different notions of distributional chaos, Li-Yorke chaos and irregularity. We thank the referee whose suggestions produced an improvement in the presentation of the paper. ${ }^{1}$
\end{abstract}

\section{Introduction}

Let us recall that an operator $T$ on a Banach space $X$ is said to be Li-Yorke chaotic if there exists an uncountable set $\Gamma \subset X$ such that for every pair $(x, y) \in \Gamma \times \Gamma$ of distinct points, we have

$$
\liminf _{n \rightarrow \infty}\left\|T^{n} x-T^{n} y\right\|=0 \text { and } \quad \limsup _{n \rightarrow \infty}\left\|T^{n} x-T^{n} y\right\|>0 .
$$

In this case, $\Gamma$ is called a scrambled set for $T$ and each such pair $(x, y)$ is called a $L i$-Yorke pair for $T$. This notion of chaos was introduced by Li and Yorke [31] in the context of interval maps. It was the first notion of chaos in the mathematical literature and it became very popular. For a study of Li-Yorke chaos and some of its variations in the context of linear dynamics, we refer the reader to the papers [13, 15, 41, 42] and references therein.

Schweizer and Smítal [36] introduced the notion of distributional chaos for interval maps as a natural strengthening of Li-Yorke chaos. Subsequently, several variations of the concept of distributional chaos were introduced and studied by various authors (see, e.g., [4, 23, 35, 37]), which we adapt here for linear operators. In order to give the precise definitions in the context of linear dynamics, let us recall that the lower and the upper densities of a set $A \subset \mathbb{N}$ are defined as

$$
\underline{\operatorname{dens}}(A):=\liminf _{n \rightarrow \infty} \frac{\operatorname{card}(A \cap[1, n])}{n} \text { and } \overline{\operatorname{dens}}(A):=\limsup _{n \rightarrow \infty} \frac{\operatorname{card}(A \cap[1, n])}{n},
$$

\footnotetext{
${ }^{1} 2010$ Mathematics Subject Classification: 47A16, 37D45.

Keywords: distributional chaos, hypercyclicity, upper-frequent hypercyclicity, distributionally irregular vector, absolutely mean irregular vector.
} 
respectively. Given an operator $T$ on a Banach space $X, x, y \in X$ and $\delta>0$, let

$$
F_{x, y}(\delta):=\underline{\operatorname{dens}}\left(\left\{j \in \mathbb{N}:\left\|T^{j} x-T^{j} y\right\|<\delta\right\}\right)
$$

and

$$
F_{x, y}^{*}(\delta):=\overline{\operatorname{dens}}\left(\left\{j \in \mathbb{N}:\left\|T^{j} x-T^{j} y\right\|<\delta\right\}\right) .
$$

Note that both $F_{x, y}$ and $F_{x, y}^{*}$ are nondecreasing maps on $(0, \infty)$ and $0 \leq F_{x, y} \leq F_{x, y}^{*} \leq 1$. Moreover,

$$
F_{x, y}(\delta)=1-\overline{\operatorname{dens}}\left(\left\{j \in \mathbb{N}:\left\|T^{j} x-T^{j} y\right\| \geq \delta\right\}\right) .
$$

The functions $F_{x, y}$ and $F_{x, y}^{*}$ are called the lower and the upper distributional functions of $x, y$ associated to $T$, respectively. If it is necessary to make clear the operator $T$ in question, we write $F_{T, x, y}$ and $F_{T, x, y}^{*}$. If the pair $(x, y)$ satisfies

(DC1) $F_{x, y}^{*} \equiv 1$ and $F_{x, y}(\varepsilon)=0$ for some $\varepsilon>0$, or

(DC2) $F_{x, y}^{*} \equiv 1$ and $F_{x, y}(\varepsilon)<1$ for some $\varepsilon>0$, or

(DC2 $\left.\frac{1}{2}\right)$ There exist $c>0$ and $r>0$ such that $F_{x, y}(\delta)<c<F_{x, y}^{*}(\delta)$ for all $0<\delta<r$, or

(DC3) $F_{x, y}(\delta)<F_{x, y}^{*}(\delta)$ for all $\delta$ in a nondegenerate interval $J \subset(0, \infty)$,

then $(x, y)$ is called a distributionally chaotic pair of type $k \in\left\{1,2,2 \frac{1}{2}, 3\right\}$ for $T$. The operator $T$ is said to be distributionally chaotic of type $k$ (DCk) if there exists an uncountable set $\Gamma \subset X$ such that every pair $(x, y)$ of distinct points in $\Gamma$ is a distributionally chaotic pair of type $k$ for $T$. In this case, $\Gamma$ is a distributionally scrambled set of type $k$ for $T$. For a study of distributional chaos in the context of linear dynamics, see $[9,13,14,24,25,32,33,38,40]$ and references therein. We observe that in these papers it is considered the following notion of distributional chaos: $T$ is said to be distributionally chaotic if there exist an uncountable set $\Gamma \subset X$ and an $\varepsilon>0$ such that for every pair $(x, y)$ of distinct points in $\Gamma$, we have that $F_{x, y}^{*} \equiv 1$ and $F_{x, y}(\varepsilon)=0$. One could call this notion of chaos by uniform distributional chaos, because the number $\varepsilon$ that appears in its definition does not depend on the pair $(x, y)$. It is obviously stronger than the notion of distributional chaos of type 1 , but they are actually equivalent for operators on Banach (or Fréchet) spaces, as follows immediately from [14, Theorem 12].

By following [14, Definition 1], we say that an operator $T$ on a Banach space $X$ is densely distributionally chaotic if there exist an uncountable dense set $\Gamma \subset X$ and an $\varepsilon>0$ such that $F_{x, y}^{*} \equiv 1$ and $F_{x, y}(\varepsilon)=0$ for every pair $(x, y)$ of distinct points in $\Gamma$.

Our goal in this paper is to investigate the above notions of distributional chaos for operators on Banach spaces, which complements and extends previous works by several authors. The paper is organized as follows.

In Section 2 we present some notations and terminologies necessary for the paper which complement those given in the Introduction.

Section 3 is devoted to the equivalence of DC1 and DC2 for operators and for $C_{0^{-}}$ semigroups of operators on Banach spaces (Theorems 2 and 6 ).

We show in Section 4 that, under certain conditions, DC $2 \frac{1}{2}$ coincides with DC1 for operators and for $C_{0}$-semigroups of operators on Banach spaces (Theorems 8 and 10 ). We also prove that every upper-frequently hypercyclic operator is DC2 $\frac{1}{2}$ (Theorem 13 . As a consequence, we extend to upper-frequently hypercyclic operators a result originally shown in [9] for frequently hypercyclic operators (Corollary 15). 
The notions of absolutely mean irregular vector and absolutely Cesàro bounded operator are considered in Section 5. In recent years, it has become very popular in the area of dynamical systems to investigate properties related to averages involving orbits or pseudo-orbits; see [27, 30, 39], where further references can be found. In our context, it is natural to ask whether or not DC1 is equivalent to the existence of an absolutely mean irregular vector. We negatively answer this question by exhibiting counterexamples on the Banach spaces $c_{0}$ and $\ell^{p}$ (Theorem 25 and Remark 26). On the other hand, sufficient conditions for the existence of a residual set of absolutely mean irregular vectors are also provided (Theorems 27 and 28). Moreover, we show that a DC2 $\frac{1}{2}$ operator cannot be absolutely Cesàro bounded (Corollary 21), but there exist operators that are mixing and absolutely Cesàro bounded (Example 23).

Section 6 contains a sufficient condition for dense distributional chaos (Theorem 33) which improves a result from [14] in the case of Banach spaces. Several consequences are presented (Corollaries 3436 and 38 41). In particular, we solve in the affirmative a conjecture of [17] (Corollary 39]).

Finally, we deal with the weakest among all the versions of distributional chaos, namely, distributional chaos of type 3 (DC3) in Section 7. Some basic properties of DC3 operators are provided (Proposition 42) and, under the condition that there exists a dense set $X_{0} \subset X$ such that $T^{n} x \rightarrow 0$ for any $x \in X_{0}$, all versions of distributional chaos are shown to be equivalent (Theorem 45). Moreover, we give examples of DC3 operators which are not Li-Yorke chaotic (Example 44) and of mixing operators which are not DC3 (Example 48). We also give examples of invertible DC1 operators $T$ such that $T^{-1}$ is not even DC3 (Example 49).

\section{Notations and preliminaries}

Throughout this paper, $X$ will denote a Banach space and $B(X)$ will denote the space of all bounded linear operators $T: X \rightarrow X$.

Let $T \in B(X)$ and $x \in X$. The orbit of $x$ is said to be distributionally near to 0 if there exists $A \subset \mathbb{N}$ with $\overline{\operatorname{dens}}(A)=1$ such that $\lim _{n \in A} T^{n} x=0$. We say that $x$ has a distributionally unbounded orbit if there exists $B \subset \mathbb{N}$ with $\overline{\operatorname{dens}}(B)=1$ such that $\lim _{n \in B}\left\|T^{n} x\right\|=\infty$. If the orbit of $x$ is simultaneously distributionally near to 0 and distributionally unbounded, then $x$ is called a distributionally irregular vector for $T$. It was proved in [14] that $T$ is uniformly distributionally chaotic if and only if $T$ admits a distributionally irregular vector. A distributionally irregular manifold for $T$ is a vector subspace $Y$ of $X$ such that every non-zero vector $y$ in $Y$ is distributionally irregular for $T$.

If $A \subset \mathbb{N}$, recall that the Banach upper density of $A$ is given by

$$
\overline{\operatorname{Bd}}(A):=\lim _{n \rightarrow \infty} \limsup _{m \rightarrow \infty} \frac{\operatorname{card}(A \cap[m+1, m+n])}{n} .
$$

Let us also recall that an operator $T \in B(X)$ is said to be frequently hypercyclic $(\mathrm{FH})$, upper-frequently hypercyclic $(\mathrm{UFH})$, reiteratively hypercyclic $(\mathrm{RH})$ or hypercyclic $(\mathrm{H})$ if there exists a point $x \in X$ such that for every nonempty open subset $U$ of $X$, the set

$$
\left\{n \in \mathbb{N}: T^{n} x \in U\right\}
$$


has positive lower density, has positive upper density, has positive upper Banach density or is nonempty, respectively. Such a point $x$ is called a frequently hypercyclic, upperfrequently hypercyclic, reiteratively hypercyclic or hypercyclic vector for $T$, respectively. Moreover, $T$ is mixing if for every nonempty open sets $U, V \subset X$, there exists $n_{0} \in \mathbb{N}$ such that $T^{n}(U) \cap V \neq \emptyset$ for all $n \geq n_{0}, T$ is weakly-mixing if $T \oplus T$ is hypercyclic, and $T$ is Devaney chaotic if it is hypercyclic and has a dense set of periodic points. See [6, 8, 16, 18, 22, for instance.

There is a useful criterion for frequent hypercyclicity, known as the Frequent Hypercyclicity Criterion (FHC) [22, Theorem 9.9]. It not only implies frequent hypercyclicity, but it also implies Devaney chaos, mixing and dense distributional chaos [14, 22].

If $A$ is a Lebesgue measurable subset of $[0, \infty)$, recall that its lower and upper densities are defined as

$$
\underline{\operatorname{Dens}}(A):=\liminf _{t \rightarrow \infty} \frac{\mu(A \cap[0, t])}{t} \text { and } \overline{\operatorname{Dens}}(A):=\limsup _{t \rightarrow \infty} \frac{\mu(A \cap[0, t])}{t},
$$

respectively, where $\mu$ denotes one-dimensional Lebesgue measure.

Given a $C_{0}$-semigroup $\mathcal{T}=\left\{T_{t}\right\}_{t \geq 0}$ of operators on $X, x, y \in X$ and $\delta>0$, the lower and the upper distributional functions of $x, y$ associated to $\mathcal{T}$ are defined by

$$
F_{\mathcal{T}, x, y}(\delta):=\underline{\operatorname{Dens}}\left(\left\{t \geq 0:\left\|T_{t} x-T_{t} y\right\|<\delta\right\}\right)
$$

and

$$
F_{\mathcal{T}, x, y}^{*}(\delta):=\overline{\operatorname{Dens}}\left(\left\{t \geq 0:\left\|T_{t} x-T_{t} y\right\|<\delta\right\}\right),
$$

respectively. Note that both $F_{\mathcal{T}, x, y}$ and $F_{\mathcal{T}, x, y}^{*}$ are nondecreasing functions on $(0, \infty)$ and $0 \leq F_{\mathcal{T}, x, y} \leq F_{\mathcal{T}, x, y}^{*} \leq 1$. Moreover

$$
F_{\mathcal{T}, x, y}(\delta)=1-\overline{\operatorname{Dens}}\left(\left\{t \geq 0:\left\|T_{t} x-T_{t} y\right\| \geq \delta\right\}\right) .
$$

Whenever the semigroup $\mathcal{T}$ is clear from the context, we write simply $F_{x, y}$ and $F_{x, y}^{*}$. We define the notions of distributional chaos of type $k \in\left\{1,2,2 \frac{1}{2}, 3\right\}$ for semigroups as we did in the case of operators, but with the distributional functions given by (3) and (4) instead of (1) and (2).

We can also define the notion of uniform distributional chaos for semigroups of operators in the obvious way. Nevertheless, as in the case of a single operator, it turns out that uniform distributional chaos and distributional chaos of type 1 coincide for $C_{0}$-semigroups of operators on Banach spaces. This will follow from Theorem 5, [1, Theorem 3.1] and the corresponding result for operators.

We refer the reader to the book [19] for a detailed study of $C_{0}$-semigroups of operators.

\section{The equivalence DC1 $\equiv$ DC2 for operators and for semigroups of operators on Banach spaces}

Let us begin this section by recalling the following definition from [14.

Definition 1. Let $T \in B(X)$. We say that $T$ satisfies the Distributional Chaos Criterion (DCC) in Banach spaces if there exist sequences $\left(x_{k}\right),\left(y_{k}\right)$ in $X$ such that:

(a) There exists $A \subset \mathbb{N}$ with $\overline{\operatorname{dens}}(A)=1$ such that $\lim _{n \in A} T^{n} x_{k}=0$ for all $k$. 
(b) $y_{k} \in \overline{\operatorname{span}\left\{x_{n}: n \in \mathbb{N}\right\}},\left\|y_{k}\right\| \rightarrow 0$ and there exist $\varepsilon>0$ and an increasing sequence $\left(N_{k}\right)$ in $\mathbb{N}$ such that

$$
\operatorname{card}\left(\left\{1 \leq j \leq N_{k}:\left\|T^{j} y_{k}\right\|>\varepsilon\right\}\right) \geq \varepsilon N_{k}
$$

for all $k \in \mathbb{N}$.

It was proved in 14 that the DCC is equivalent to DC1 for operators on Banach spaces. Let us now prove that it is also equivalent to DC2.

Theorem 2. For every $T \in B(X)$, the following assertions are equivalent:

(i) T satisfies the DCC in Banach spaces;

(ii) T admits a distributionally irregular vector;

(iii) $T$ is distributionally chaotic of type 1 ;

(iv) $T$ admits a distributionally chaotic pair of type 1 ;

(v) $T$ is distributionally chaotic of type 2 ;

(vi) T admits a distributionally chaotic pair of type 2 .

Proof. (i) $\Leftrightarrow$ (ii) $\Leftrightarrow$ (iii) $\Leftrightarrow$ (iv): [14, Theorem 12].

(iii) $\Rightarrow(\mathrm{v}) \Rightarrow$ (vi): Obvious.

(vi) $\Rightarrow$ (i): Since $T$ admits a distributionally chaotic pair of type 2 , there exist $u \in X$ and $\zeta>0$ such that

$$
\xi:=\overline{\operatorname{dens}}\left(\left\{j \in \mathbb{N}:\left\|T^{j} u\right\|>\zeta\right\}\right)>0
$$

and

$$
\overline{\operatorname{dens}}\left(\left\{j \in \mathbb{N}:\left\|T^{j} u\right\|<\delta\right\}\right)=1,
$$

for all $\delta>0$. By $(6)$, there exists an increasing sequence $\left(n_{k}\right)$ in $\mathbb{N}$ such that

$$
\frac{\operatorname{card}\left(\left\{1 \leq j \leq n_{k}:\left\|T^{j} u\right\|<1 / k\right\}\right)}{n_{k}}>1-\frac{1}{k} .
$$

Hence, the set

$$
A:=\bigcup_{k=1}^{\infty}\left\{1 \leq j \leq n_{k}:\left\|T^{j} u\right\|<1 / k\right\}
$$

satisfies $\overline{\operatorname{dens}}(A)=1$. Let $x_{k}:=T^{k} u(k \in \mathbb{N})$. Then

$$
\lim _{n \in A} T^{n} x_{k}=0 \quad \text { for all } k \in \mathbb{N} \text {. }
$$

For each $k \in \mathbb{N}$, choose $m_{k} \in \mathbb{N}$ such that $\left\|T^{m_{k}} u\right\|<1 / k$, and define $y_{k}:=T^{m_{k}} u$. Then $y_{k} \in \overline{\operatorname{span}\left\{x_{n}: n \in \mathbb{N}\right\}}$ and $\left\|y_{k}\right\| \rightarrow 0$. Let $\varepsilon:=\min \left\{\zeta, \frac{\xi}{2}\right\}$. By (5),

$$
\overline{\operatorname{dens}}\left(\left\{j \in \mathbb{N}:\left\|T^{j} y_{k}\right\|>\varepsilon\right\}\right) \geq \xi>\varepsilon \quad(k \in \mathbb{N}) .
$$

So, there exists an increasing sequence $\left(N_{k}\right)$ in $\mathbb{N}$ such that

$$
\frac{\operatorname{card}\left(\left\{1 \leq j \leq N_{k}:\left\|T^{j} y_{k}\right\|>\varepsilon\right\}\right)}{N_{k}}>\varepsilon \quad(k \in \mathbb{N}) .
$$

This proves that $T$ satisfies the DCC in Banach spaces. 
Let us now consider the case of semigroups of operators. For this purpose, we shall need the following result, which is a slight variation of Lemma 2.4 in [1].

Lemma 3. Let $\mathcal{T}:=\left\{T_{t}\right\}_{t \geq 0}$ be a $C_{0}$-semigroup of operators on $X, s>0$ and $x \in X$. Put $C_{s}:=\sup _{0 \leq t \leq s}\left\|T_{t}\right\|$. Then, for every $\delta>0$, we have that:

(a) $\underline{\operatorname{Dens}}\left(\left\{t \geq 0:\left\|T_{t} x\right\|<\delta\right\}\right) \leq \underline{\operatorname{dens}}\left(\left\{j \in \mathbb{N}:\left\|T_{s}^{j} x\right\|<C_{s} \delta\right\}\right)$.

(b) $\underline{\operatorname{dens}}\left(\left\{j \in \mathbb{N}:\left\|T_{s}^{j} x\right\|<\delta\right\}\right) \leq \underline{\operatorname{Dens}}\left(\left\{t \geq 0:\left\|T_{t} x\right\|<C_{s} \delta\right\}\right)$.

(c) $\overline{\operatorname{Dens}}\left(\left\{t \geq 0:\left\|T_{t} x\right\|<\delta\right\}\right) \leq \overline{\operatorname{dens}}\left(\left\{j \in \mathbb{N}:\left\|T_{s}^{j} x\right\|<C_{s} \delta\right\}\right)$.

(d) $\overline{\operatorname{dens}}\left(\left\{j \in \mathbb{N}:\left\|T_{s}^{j} x\right\|<\delta\right\}\right) \leq \overline{\operatorname{Dens}}\left(\left\{t \geq 0:\left\|T_{t} x\right\|<C_{s} \delta\right\}\right)$.

Items (c) and (d) are exactly items (3') and (4') in [1, Lemma 2.4], while items (a) and (b) are slight variations of items (2') and (1') in this lemma. However, we shall give a proof of items (a) and (b) for the sake of completeness.

Proof. (a) Put $A:=\left\{t \geq 0:\left\|T_{t} x\right\|<\delta\right\}$ and $B:=\left\{j \in \mathbb{N}:\left\|T_{s}^{j} x\right\|<C_{s} \delta\right\}$. Let $n \in \mathbb{N}$. If $j \in \mathbb{N}$ and $A \cap[(j-1) s, j s] \neq \emptyset$, then $j \in B$. Indeed, let $t \in A \cap[(j-1) s, j s]$ and write $t=(j-1) s+r$ with $0 \leq r \leq s$. Then

$$
\left\|T_{s}^{j} x\right\|=\left\|T_{t+s-r} x\right\| \leq\left\|T_{s-r}\right\|\left\|T_{t} x\right\|<C_{s} \delta,
$$

proving that $j \in B$. Therefore,

$$
\frac{\mu(A \cap[0, n s])}{n s}=\frac{1}{n} \sum_{j=1}^{n} \frac{\mu(A \cap[(j-1) s, j s])}{s} \leq \frac{\operatorname{card}(B \cap[1, n])}{n} .
$$

This implies the inequality in (a).

(b) Put $A:=\left\{j \in \mathbb{N}:\left\|T_{s}^{j} x\right\|<\delta\right\}$ and $B:=\left\{t \geq 0:\left\|T_{t} x\right\|<C_{s} \delta\right\}$. If $j \in A$, then

$$
\left\|T_{j s+r} x\right\| \leq\left\|T_{r}\right\|\left\|T_{j s} x\right\|<C_{s} \delta \quad(0 \leq r \leq s),
$$

that is, $[j s,(j+1) s] \subset B$. Hence, for all $n \in \mathbb{N}$,

$$
\frac{\operatorname{card}(A \cap[1, n])}{n} \leq \frac{1}{n} \sum_{j=1}^{n} \frac{\mu(B \cap[j s,(j+1) s])}{s}=\frac{\mu(B \cap[s,(n+1) s])}{n s},
$$

which implies the inequality in (b).

Lemma 4. Let $\mathcal{T}:=\left\{T_{t}\right\}_{t \geq 0}$ be a $C_{0}$-semigroup of operators on $X, s>0$ and $x, y \in X$. Put $C_{s}:=\sup _{0 \leq t \leq s}\left\|T_{t}\right\|$. Then, for every $\delta>0$, we have that:

(a) $F_{\mathcal{T}, x, y}(\delta) \leq F_{T_{s}, x, y}\left(C_{s} \delta\right)$.

(b) $F_{T_{s}, x, y}(\delta) \leq F_{\mathcal{T}, x, y}\left(C_{s} \delta\right)$.

(c) $F_{\mathcal{T}, x, y}^{*}(\delta) \leq F_{T_{s}, x, y}^{*}\left(C_{s} \delta\right)$.

(d) $F_{T_{s}, x, y}^{*}(\delta) \leq F_{\mathcal{T}, x, y}^{*}\left(C_{s} \delta\right)$. 
Proof. (a) By Lemma 3(a),

$$
\begin{aligned}
F_{\mathcal{T}, x, y}(\delta) & =\underline{\operatorname{Dens}}\left(\left\{t \geq 0:\left\|T_{t} x-T_{t} y\right\|<\delta\right\}\right) \\
& \leq \underline{\operatorname{dens}}\left(\left\{j \in \mathbb{N}:\left\|T_{s}^{j} x-T_{s}^{j} y\right\|<C_{s} \delta\right\}\right) \\
& =F_{T_{s}, x, y}\left(C_{s} \delta\right) .
\end{aligned}
$$

The proofs of the other items are analogous.

Theorem 5. For every $C_{0}$-semigroup $\mathcal{T}:=\left\{T_{t}\right\}_{t \geq 0}$ of operators on $X$, the following assertions are equivalent:

(i) $\mathcal{T}$ is $D C 1$ (resp. DCQ);

(ii) $T_{s}$ is DC1 (resp. DC2) for some $s>0$;

(iii) $T_{s}$ is DC1 (resp. DC2) for every $s>0$.

Proof. Given a pair $(x, y)$ of points in $X$, it follows from Lemma 4 that the following assertions are equivalent:

- $(x, y)$ is a distributionally chaotic pair of type 1 (resp. 2) for $\mathcal{T}$;

- $(x, y)$ is a distributionally chaotic pair of type 1 (resp. 2) for $T_{s}$ for some $s>0$;

- $(x, y)$ is a distributionally chaotic pair of type 1 (resp. 2) for $T_{s}$ for every $s>0$.

This clearly implies the theorem.

By combining Theorems 2 and 5 , we obtain the following.

Theorem 6. $A C_{0}$-semigroup $\mathcal{T}$ of operators on $X$ is DC1 if and only if it is DC2.

\section{$4 \quad \mathrm{DC} 2 \frac{1}{2}$ in Banach spaces}

Lemma 7. Let $T \in B(X)$. If $T$ admits a distributionally chaotic pair of type $2 \frac{1}{2}$, then $T$ admits a distributionally unbounded orbit.

Proof. Since $T$ admits a distributionally chaotic pair of type $2 \frac{1}{2}$, there exist $u \in X, c>0$ and $\zeta>0$ such that

$$
\xi:=\overline{\operatorname{dens}}\left(\left\{j \in \mathbb{N}:\left\|T^{j} u\right\|>\zeta\right\}\right)>0
$$

and

$$
\overline{\operatorname{dens}}\left(\left\{j \in \mathbb{N}:\left\|T^{j} u\right\|<\delta\right\}\right)>c,
$$

for all $\delta>0$. Thus, there exists an increasing sequence $\left(n_{k}\right)$ in $\mathbb{N}$ such that $\left\|T^{n_{k}} u\right\|<1 / k$ for all $k \in \mathbb{N}$. Let $y_{k}:=T^{n_{k}} u$ and $\varepsilon:=\min \left\{\zeta, \frac{\xi}{2}\right\}$. Then $\lim _{k \rightarrow \infty} y_{k}=0$ and

$$
\overline{\operatorname{dens}}\left(\left\{j \in \mathbb{N}:\left\|T^{j} y_{k}\right\|>\zeta\right\}\right)=\xi>\varepsilon \quad(k \in \mathbb{N}) .
$$

Hence, there exists an increasing sequence $\left(N_{k}\right)$ in $\mathbb{N}$ such that

$$
\operatorname{card}\left(\left\{1 \leq j \leq N_{k}:\left\|T^{j} y_{k}\right\|>\varepsilon\right\}\right) \geq \varepsilon N_{k},
$$

for all $k \in \mathbb{N}$. Therefore, by [14, Proposition 8], there exists $y \in X$ with distributionally unbounded orbit. 
Now we will see that under the condition that there exists a dense set $X_{0} \subset X$ such that $T^{n} x \rightarrow 0$ for all $x \in X_{0}$, DC2 $\frac{1}{2}$ is also equivalent to DC1.

Theorem 8. Let $T \in B(X)$ and assume that there exists a dense set $X_{0} \subset X$ such that

$$
T^{n} x \rightarrow 0 \quad \text { for all } x \in X_{0} .
$$

Then the following assertions are equivalent:

(i) T satisfies the DCC in Banach spaces;

(ii) T admits a distributionally irregular vector;

(iii) $T$ is distributionally chaotic of type 1 ;

(iv) $T$ is distributionally chaotic of type 2 ;

(v) $T$ is distributionally chaotic of type $2 \frac{1}{2}$;

(vi) T admits a distributionally chaotic pair of type $2 \frac{1}{2}$.

Proof. It is enough to prove that (vi) $\Rightarrow$ (ii). So, assume (vi). The proof of $[14$, Theorem 15] shows that if an operator $T$ has a distributionally unbounded orbit and $T^{n} x \rightarrow 0$ for every $x$ in a dense subset of $X$, then $T$ has a distributionally irregular vector. Hence, in view of our hypotheses and the previous lemma, we conclude that (ii) holds.

Let us now turn to semigroups of operators.

Theorem 9. For every $C_{0}$-semigroup $\mathcal{T}:=\left\{T_{t}\right\}_{t \geq 0}$ of operators on $X$, the following assertions are equivalent:

(i) $\mathcal{T}$ is $D C 2 \frac{1}{2}$;

(ii) $T_{s}$ is DC2 $\frac{1}{2}$ for some $s>0$;

(iii) $T_{s}$ is DC2 $\frac{1}{2}$ for every $s>0$.

Proof. (i) $\Rightarrow$ (iii): Let $c>0$ and $r>0$ be such that $F_{\mathcal{T}, x, y}(\delta)<c<F_{\mathcal{T}, x, y}^{*}(\delta)$ for all $0<\delta<r$. By Lemma 4 (b), (c),

$$
F_{T_{s}, x, y}(\delta) \leq F_{\mathcal{T}, x, y}\left(C_{s} \delta\right)<c \quad \text { whenever } 0<\delta<r / C_{s}
$$

and

$$
F_{T_{s}, x, y}^{*}(\delta) \geq F_{\mathcal{T}, x, y}^{*}\left(\delta / C_{s}\right)>c \quad \text { whenever } 0<\delta<C_{s} r .
$$

Hence, $F_{T_{s}, x, y}(\delta)<c<F_{T_{s}, x, y}^{*}(\delta)$ for all $0<\delta<r / C_{s}$.

(iii) $\Rightarrow$ (ii): Obvious.

(ii) $\Rightarrow$ (i): If $F_{T_{s}, x, y}(\delta)<c<F_{T_{s}, x, y}^{*}(\delta)$ for all $0<\delta<r$, it follows from Lemma 4(a),(d) that $F_{\mathcal{T}, x, y}(\delta)<c<F_{\mathcal{T}, x, y}^{*}(\delta)$ for all $0<\delta<r / C_{s}$.

By combining Theorems 5, 8 and 9 we obtain the following.

Theorem 10. Suppose that $\mathcal{T}:=\left\{T_{t}\right\}_{t \geq 0}$ is a $C_{0}$-semigroup of operators on $X$ such that

$$
\lim _{t \rightarrow \infty} T_{t} x=0 \quad \text { for all } x \in X_{0}
$$

where $X_{0}$ is a dense subset of $X$. Then $\mathcal{T}$ is DC2 $\frac{1}{2}$ if and only if $\mathcal{T}$ is DC1. 
Corollary 11. Let $1 \leq p<\infty$ and let $v: \mathbb{R}_{+} \rightarrow \mathbb{R}$ be a strictly positive locally integrable function for which there exist $M \geq 1$ and $w \in \mathbb{R}$ such that, for all $t \geq 0$,

$$
v(x) \leq M e^{w t} v(x+t) \quad \text { for almost all } x \geq 0 .
$$

Consider the translation semigroup $\mathcal{T}$ on the space of weighted p-integrable functions $L_{v}^{p}\left(\mathbb{R}_{+}\right)$([22], Example 7.4). Then $\mathcal{T}$ is DC2 $\frac{1}{2}$ if and only if $\mathcal{T}$ is DC1.

Proof. Since the orbit of any continuous function of compact support converges to zero, we can apply the above theorem.

Bayart and Ruzsa [9] proved that if $T \in B(X)$ is frequently hypercyclic and there exists a dense set $X_{0} \subset X$ such that $T^{n} x \rightarrow 0$ for any $x \in X_{0}$, then $T$ is DC1.

Our next goal is to prove that every upper-frequently hypercyclic operator (and, in particular, every frequently hypercyclic operator) is DC $2 \frac{1}{2}$. As a consequence, we will extend the above-mentioned result from [9] to upper-frequently hypercyclic operators.

Lemma 12. Let $T \in B(X)$. If $T$ is upper-frequently hypercyclic, then $T$ admits a distributionally unbounded orbit.

Proof. Since $T$ is upper-frequently hypercyclic, there exist $x \in X$ and an increasing sequence $\left(n_{k}\right)$ in $\mathbb{N}$ such that

$$
\xi:=\overline{\operatorname{dens}}\left(\left\{j \in \mathbb{N}:\left\|T^{j} x\right\|>1\right\}\right)>0
$$

and

$$
\left\|T^{n_{k}} x\right\|<\frac{1}{k} \quad \text { for all } k \in \mathbb{N} .
$$

Define $y_{k}:=T^{n_{k}} x$ and $\varepsilon:=\xi / 2$. Then $\lim _{k \rightarrow \infty} y_{k}=0$ and

$$
\overline{\operatorname{dens}}\left(\left\{j \in \mathbb{N}:\left\|T^{j} y_{k}\right\|>1\right\}\right)=\xi>\varepsilon \quad(k \in \mathbb{N}) .
$$

Hence, there exists an increasing sequence $\left(N_{k}\right)$ in $\mathbb{N}$ such that

$$
\operatorname{card}\left(\left\{1 \leq j \leq N_{k}:\left\|T^{j} y_{k}\right\|>\varepsilon\right\}\right) \geq \varepsilon N_{k},
$$

for all $k \in \mathbb{N}$. Therefore, by [14, Proposition 8], there exists $y \in X$ with distributionally unbounded orbit.

Theorem 13. Let $T \in B(X)$. If $T$ is upper-frequently hypercyclic, then $T$ is distributionally chaotic of type $2 \frac{1}{2}$.

Proof. By Lemma 12 and [14, Proposition 8], there exists a residual set $R_{1}$ of vectors $x \in X$ such that $\left\|T^{j} x\right\| \rightarrow \infty$ as $j \rightarrow \infty$ along some set $E_{x}$ with $\overline{\operatorname{dens}}\left(E_{x}\right)=1$.

As consequence of [21, Proposition 4.7], there exists a residual set $R_{2}$ of vectors $y \in X$ such that $\left\|T^{j} y\right\| \rightarrow 0$ as $j \rightarrow \infty$ along some set $E_{y}$ with $\overline{\operatorname{dens}}\left(E_{y}\right) \geq C>0$.

Let $z \in R_{1} \cap R_{2}$. Then the set $\{\alpha z: \alpha \in \mathbb{K}\}$ is an uncountable distributionally scrambled set of type $2 \frac{1}{2}$ for $T$. Thus $T$ is distributionally chaotic of type $2 \frac{1}{2}$.

Corollary 14. If $T \in B(X)$ is frequently hypercyclic and invertible, then both $T$ and $T^{-1}$ are distributionally chaotic of type $2 \frac{1}{2}$.

Proof. By [9, Proposition 20], both $T$ and $T^{-1}$ are upper-frequently hypercyclic. Hence, we can apply the above theorem. 
Corollary 15. If $T \in B(X)$ is upper-frequently hypercyclic and there exists a dense set $X_{0} \subset X$ such that $T^{n} x \rightarrow 0$ for any $x \in X_{0}$, then $T$ is distributionally chaotic of type 1 .

Proof. By the above theorem, $T$ is distributionally chaotic of type $2 \frac{1}{2}$. Since there exists a dense set $X_{0} \subset X$ such that $T^{n} x \rightarrow 0$ for any $x \in X_{0}$, Theorem 8 implies that $T$ is distributionally chaotic of type 1 .

Remark 16. Bayart and Ruzsa [9, Section 6] proved that there exists a frequently hypercyclic operator which is not distributionally chaotic. Thus, this operator is DC2 $\frac{1}{2}$ and not DC1.

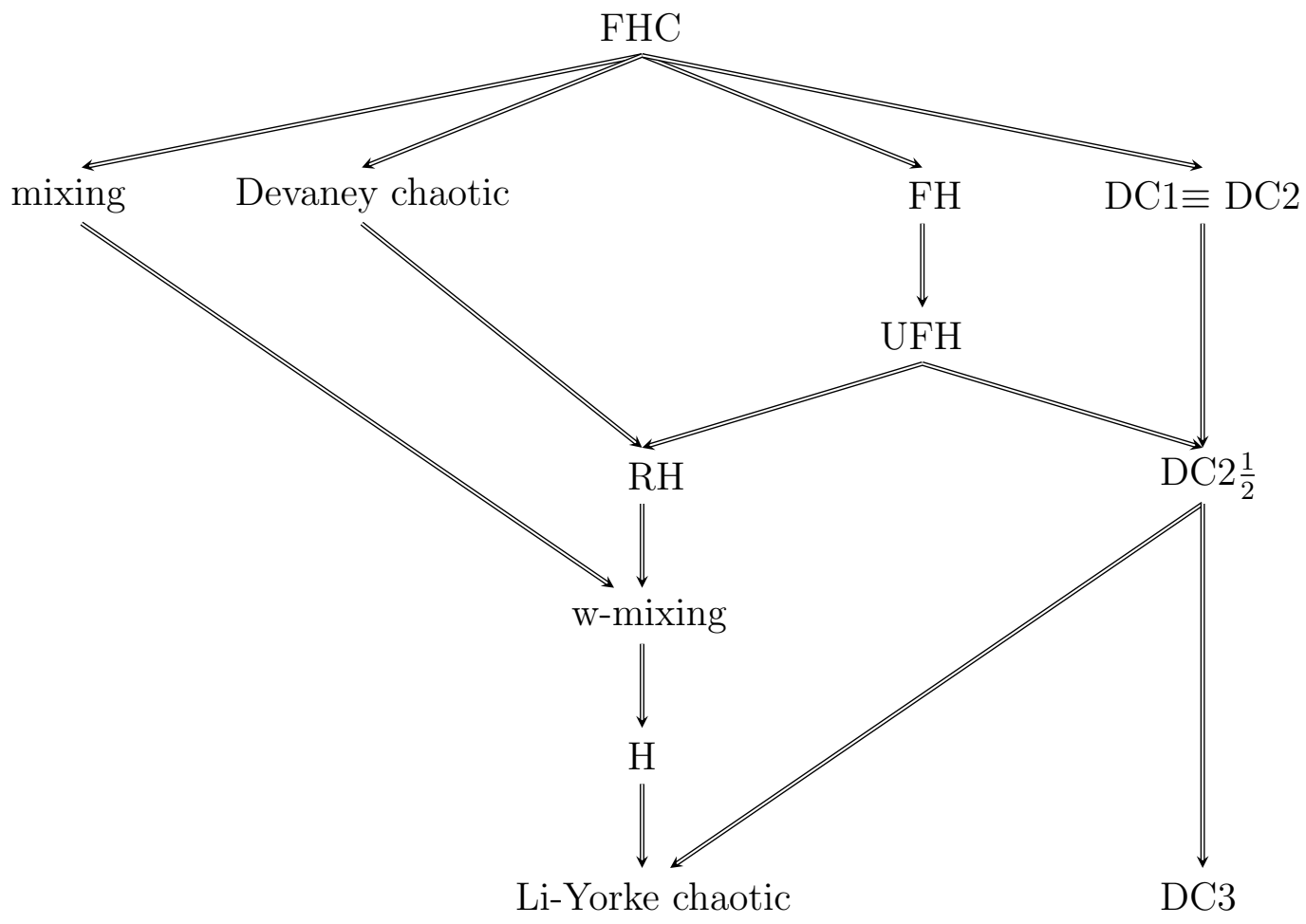

Figure 1: Implications between different definitions related with hypercyclicity and chaos in Banach spaces.

Menet [34] proved that there exists a Devaney chaotic operator on $l^{1}(\mathbb{N})$ which is not DC1 and is not upper-frequently hypercyclic. Since both concepts imply DC $2 \frac{1}{2}$, it is natural to consider the following question.

Problem 17. Is there a Devaney chaotic operator on a Banach space which is not DC $2 \frac{1}{2}$ ?

\section{Distributional chaos and absolutely mean irregular vectors}

We begin this section with two definitions.

Definition 18. Given $T \in B(X)$ and $x \in X$, we say that $x$ is an absolutely mean irregular vector for $T$ if

$$
\liminf _{N \rightarrow \infty} \frac{1}{N} \sum_{j=1}^{N}\left\|T^{j} x\right\|=0 \quad \text { and } \quad \limsup _{N \rightarrow \infty} \frac{1}{N} \sum_{j=1}^{N}\left\|T^{j} x\right\|=\infty .
$$


Definition 19 ([25]). An operator $T \in B(X)$ is said to be absolutely Cesàro bounded if there exists a constant $C>0$ such that

$$
\sup _{N \in \mathbb{N}} \frac{1}{N} \sum_{j=1}^{N}\left\|T^{j} x\right\| \leq C\|x\| \quad \text { for all } x \in X .
$$

The following result was proved in [25]: If $T$ is DC2 and $\left\{x \in X: \lim _{n \rightarrow \infty}\left\|T^{n} x\right\|=0\right\}$ is dense in $X$, then $T$ is not absolutely Cesàro bounded.

The next proposition shows that there are some relations between the averages

$$
\frac{1}{N} \sum_{j=1}^{N}\left\|T^{j} x\right\|
$$

and the notions of distributionally near to 0 and distributionally unbounded orbits. It also shows that the hypothesis that the set $\left\{x \in X: \lim _{n \rightarrow \infty}\left\|T^{n} x\right\|=0\right\}$ is dense in $X$ is superfluous in the above-mentioned result from [25].

Proposition 20. For any $T \in B(X)$, we have that:

(a) If $\liminf _{N \rightarrow \infty} \frac{1}{N} \sum_{j=1}^{N}\left\|T^{j} x\right\|=0$, then the orbit of $x$ is distributionally near to 0 .

(b) If the orbit of $x$ is distributionally unbounded, then $\limsup _{N \rightarrow \infty} \frac{1}{N} \sum_{j=1}^{N}\left\|T^{j} x\right\|=\infty$.

Proof. Note that

$$
\frac{\operatorname{card}\left(\left\{1 \leq j \leq N:\left\|T^{j} x\right\| \geq \delta\right\}\right)}{N} \leq \frac{1}{N} \sum_{j=1}^{N} \frac{\left\|T^{j} x\right\|}{\delta}=\frac{1}{\delta} \frac{1}{N} \sum_{j=1}^{N}\left\|T^{j} x\right\| .
$$

(a) If $\liminf _{N \rightarrow \infty} \frac{1}{N} \sum_{j=1}^{N}\left\|T^{j} x\right\|=0$, it follows from 7 that

$$
\underline{\operatorname{dens}}\left(\left\{j \in \mathbb{N}:\left\|T^{j} x\right\| \geq \delta\right\}\right)=0 \quad \text { for all } \delta>0,
$$

and so

$$
\overline{\operatorname{dens}}\left(\left\{j \in \mathbb{N}:\left\|T^{j} x\right\|<\delta\right\}\right)=1 \text { for all } \delta>0 .
$$

This is equivalent to say that the orbit of $x$ is distributionally near to 0 .

(b) If the orbit of $x$ is distributionally unbounded, then

$$
\overline{\operatorname{dens}}\left(\left\{j \in \mathbb{N}:\left\|T^{j} x\right\| \geq k\right\}\right)=1 \quad \text { for all } k \in \mathbb{N},
$$

and so (7) implies that

$$
\limsup _{N \rightarrow \infty} \frac{1}{N} \sum_{j=1}^{N}\left\|T^{j} x\right\| \geq k \quad \text { for all } k \in \mathbb{N},
$$

which gives the desired result. 
Corollary 21. If $T$ is distributionally chaotic of type $2 \frac{1}{2}$, then $T$ is not absolutely Cesàro bounded.

Proof. By Lemma 7, T admits a distributionally unbounded orbit. Hence, Proposition 20(b) implies that $T$ is not absolutely Cesàro bounded.

Corollary 22. No upper-frequently hypercyclic operator can be absolutely Cesàro bounded.

On the other hand, a mixing operator can be absolutely Cesàro bounded.

Example 23. There exist operators $T \in B\left(\ell^{p}(\mathbb{N})\right), 1 \leq p<\infty$, that are mixing and absolutely Cesàro bounded.

(A) Let us consider the operator defined in [14, Example 5]. It is the unilateral weighted backward shift

$$
T:\left(x_{1}, x_{2}, \ldots\right) \in \ell^{1}(\mathbb{N}) \mapsto\left(w_{2} x_{2}, w_{3} x_{3}, \ldots\right) \in \ell^{1}(\mathbb{N})
$$

whose weight sequence is given by

$$
w_{k}:=\left(\frac{k}{k-1}\right)^{1-\varepsilon} \text { for } k \geq 2,
$$

where $\varepsilon \in\left(0, \frac{1}{5}\right)$ is fixed. It was proved in [14] that

$$
\frac{1}{N} \sum_{j=1}^{N}\left\|T^{j} x\right\| \leq\left(2+\frac{2}{\varepsilon}\right)\|x\| \quad \text { for all } x \in \ell^{1}(\mathbb{N}) .
$$

Hence, $T$ is absolutely Cesàro bounded. Since $\prod_{k=2}^{n} w_{k}=n^{1-\varepsilon} \rightarrow \infty$ as $n \rightarrow \infty, T$ is also mixing [22, Example 4.9].

(B) More recently, it was proved in 25] that the unilateral weighted backward shift $B_{w}: \ell^{1}(\mathbb{N}) \rightarrow \ell^{1}(\mathbb{N})$ with weights

$$
w_{k}:=\frac{2 k}{2 k-1} \quad(k \in \mathbb{N})
$$

is also mixing and absolutely Cesàro bounded.

(C) In 33 the following example was provided: Let $n_{k}:=(k !)^{3}(k \in \mathbb{N})$ and select the sequence of weights $v=\left(v_{j}\right)_{j \in \mathbb{N}}$ given by $v_{j}=1 / k$ for $n_{k} \leq j<n_{k+1}(k \in \mathbb{N})$. In [33, Theorem 2.1] it was directly shown that the unilateral backward shift $B$ on $\ell^{p}(v)(1 \leq p<\infty)$ is mixing, and that, for each $x \in \ell^{p}(v)$ and each $\varepsilon>0$,

$$
\lim _{n \rightarrow \infty} \frac{\operatorname{card}\left(\left\{1 \leq j \leq n:\left\|B^{j} x\right\|<\varepsilon\right\}\right)}{n}=1,
$$

which implies that $B$ is absolutely Cesàro bounded.

The third example (C) above was also used in the PhD Thesis of M. J. Beltrán [10] to obtain examples of uniformly mean ergodic operators that are mixing, by following an observation of the third author. See also [11, 12 for more results on this topic. We were informed by Bonet that in [2] the authors observe that there are hypercyclic weighted shifts on the Hilbert space which are Cesàro bounded.

Since reiteratively hypercyclic operators are weakly mixing, it is reasonable to make the following question. 
Problem 24. Is there a reiteratively hypercyclic operator which is absolutely Cesàro bounded?

In view of Proposition 20 and the fact that $\mathrm{DC} 1$ is characterized by the existence of a distributionally irregular vector, it is natural to ask the following question:

Is DC1 equivalent to the existence of an absolutely mean irregular vector?

Let us now see that the answer to this question is "no".

Theorem 25. If $X=c_{0}(\mathbb{N})$ or $X=\ell^{p}(\mathbb{N})$ for some $1 \leq p \leq \infty$, then there exists an operator $T \in B(X)$ which is distributionally chaotic of type 1 and satisfies

$$
\lim _{N \rightarrow \infty} \frac{1}{N} \sum_{j=1}^{N}\left\|T^{j} x\right\|=\infty \quad \text { for all } x \in X \backslash\{0\} .
$$

Proof. Our operator will be a unilateral weighted forward shift

$$
T:\left(x_{1}, x_{2}, \ldots\right) \in X \mapsto\left(0, w_{1} x_{1}, w_{2} x_{2}, \ldots\right) \in X
$$

with a suitably chosen weight sequence $w=\left(w_{n}\right)_{n \in \mathbb{N}}$. The sequence $w$ will be obtained as a sequence of blocks

$$
w:=\left(B_{1} C_{1} B_{2} C_{2} B_{3} C_{3} \ldots\right)
$$

where each $B_{n}$ has the form

$$
B_{n}:=(\underbrace{2,2, \ldots, 2}_{2 k_{n} \text { times }}, 1, \underbrace{\frac{1}{2}, \frac{1}{2}, \ldots, \frac{1}{2}}_{2 k_{n}-1 \text { times }}, \frac{n}{2(n+1)}, \underbrace{1,1, \ldots, 1}_{2^{k_{n}-1 \text { times }}})
$$

and each $C_{n}$ has the form

$$
C_{n}:=(\underbrace{2,2, \ldots, 2}_{r_{n} \text { times }}, \underbrace{1,1, \ldots, 1}_{j_{n}-1 \text { times }}, \underbrace{\frac{1}{2}, \frac{1}{2}, \ldots, \frac{1}{2}}_{r_{n} \text { times }}) .
$$

The number $r_{n}$ is the smallest positive integer greater than or equal to $2 \log _{2}(n+1)$ and the numbers $k_{n}$ and $j_{n}$ will be chosen inductively. We shall denote by $b_{n}$ (resp. $c_{n}$ ) the length of the sequence $B_{n}$ (resp. $C_{n}$ ); in other words, $b_{n}=4 k_{n}+2^{k_{n}}$ and $c_{n}=2 r_{n}+j_{n}-1$ $(n \in \mathbb{N})$. Moreover, we define $s_{n}:=b_{1}+c_{1}+b_{2}+c_{2}+\cdots+b_{n}$ and $t_{n}:=s_{n}+c_{n}(n \in \mathbb{N})$, and $t_{0}:=0$.

We start with $k_{1}:=2$ and $j_{1}:=2$, so that

$$
B_{1}=(2,2,2,2,1,1 / 2,1 / 2,1 / 2,1 / 4,1,1,1) \quad \text { and } \quad C_{1}=(2,2,1,1 / 2,1 / 2) .
$$

Suppose that $n \geq 2$ and that $k_{1}, \ldots, k_{n-1}$ and $j_{1}, \ldots, j_{n-1}$ have been chosen. Note that

$$
\left(\left\|T^{j} e_{1}\right\|\right)_{t_{n-1}<j \leq s_{n}}=(\frac{2}{n}, \frac{2^{2}}{n}, \ldots, \frac{2^{2 k_{n}}}{n}, \frac{2^{2 k_{n}}}{n}, \frac{2^{2 k_{n}-1}}{n}, \ldots, \frac{2}{n}, \underbrace{\frac{1}{n+1}, \frac{1}{n+1}, \ldots, \frac{1}{n+1}}_{2^{k_{n}} \text { times }})
$$

and

$$
\left(\left\|T^{j} e_{1}\right\|\right)_{s_{n}<j \leq t_{n}}=(\frac{2}{n+1}, \ldots, \frac{2^{r_{n}-1}}{n+1}, \underbrace{\frac{2^{r_{n}}}{n+1}, \frac{2^{r_{n}}}{n+1}, \ldots, \frac{2^{r_{n}}}{n+1}}_{j_{n} \text { times }}, \frac{2^{r_{n}-1}}{n+1}, \ldots, \frac{2}{n+1}, \frac{1}{n+1}) .
$$


Note also that

$$
\frac{2^{r_{n}}}{n+1} \geq n+1
$$

Now

$$
\frac{\operatorname{card}\left(\left\{1 \leq j \leq s_{n}:\left\|T^{j} e_{1}\right\| \leq \frac{1}{n+1}\right\}\right)}{s_{n}} \geq \frac{2^{k_{n}}}{t_{n-1}+4 k_{n}+2^{k_{n}}} \rightarrow 1 \text { as } k_{n} \rightarrow \infty
$$

Hence, we can choose $k_{n}$ large enough so that

$$
\frac{\operatorname{card}\left(\left\{1 \leq j \leq s_{n}:\left\|T^{j} e_{1}\right\| \leq \frac{1}{n+1}\right\}\right)}{s_{n}} \geq 1-\frac{1}{n} .
$$

Let

$$
A_{n}:=\frac{1}{s_{n}} \sum_{j=1}^{s_{n}}\left\|T^{j} e_{1}\right\|
$$

Since

$$
A_{n}=\frac{\sum_{j=1}^{t_{n-1}}\left\|T^{j} e_{1}\right\|+\frac{2\left(2^{2 k_{n}+1}-2\right)}{n}+\frac{2^{k_{n}}}{n+1}}{t_{n-1}+4 k_{n}+2^{k_{n}}} \geq \frac{2^{2 k_{n}+2}-4}{n\left(t_{n-1}+4 k_{n}+2^{k_{n}}\right)} \rightarrow \infty \text { as } k_{n} \rightarrow \infty,
$$

we can assume that $k_{n}$ was chosen so large that

$$
\frac{s_{n} A_{n}}{s_{n}+3 r_{n}} \geq n+1
$$

On the other hand,

$$
\frac{\operatorname{card}\left(\left\{1 \leq j \leq t_{n}:\left\|T^{j} e_{1}\right\| \geq n+1\right\}\right)}{t_{n}} \geq \frac{j_{n}}{s_{n}+2 r_{n}+j_{n}-1} \rightarrow 1 \text { as } j_{n} \rightarrow \infty
$$

Therefore, we can choose $j_{n}$ so large that

$$
\frac{\operatorname{card}\left(\left\{1 \leq j \leq t_{n}:\left\|T^{j} e_{1}\right\| \geq n+1\right\}\right)}{t_{n}} \geq 1-\frac{1}{n} .
$$

It follows from $(8)$ that $\overline{\operatorname{dens}}\left(\left\{j \in \mathbb{N}:\left\|T^{j} e_{1}\right\|<\delta\right\}\right)=1$ for all $\delta>0$, that is, the orbit of $e_{1}$ is distributionally near to 0 . And by $\sqrt{10}, \overline{\operatorname{dens}}\left(\left\{j \in \mathbb{N}:\left\|T^{j} e_{1}\right\| \geq k\right\}\right)=1$ for all $k \in \mathbb{N}$, that is, the orbit of $e_{1}$ is distributionally unbounded. Thus, $e_{1}$ is a distributionally irregular vector for $T$ and $T$ is DC1.

Finally, it follows from $(9)$ that

$$
\frac{1}{N} \sum_{j=1}^{N}\left\|T^{j} e_{1}\right\| \geq n+1 \quad \text { whenever } t_{n-1}+4 k_{n} \leq N \leq t_{n}+4 k_{n+1} .
$$

Thus,

$$
\lim _{N \rightarrow \infty} \frac{1}{N} \sum_{j=1}^{N}\left\|T^{j} x\right\|=\infty \quad \text { for all } x \neq 0,
$$

which completes the proof. 
Remark 26. We have the following variation of the previous result: If $X=c_{0}(\mathbb{Z})$ or $X=\ell^{p}(\mathbb{Z})$ for some $1 \leq p \leq \infty$, then there exists an invertible operator $T \in B(X)$ which is distributionally chaotic of type 1 and satisfies

$$
\lim _{N \rightarrow \infty} \frac{1}{N} \sum_{j=1}^{N}\left\|T^{j} x\right\|=\infty \quad \text { for all } x \in X \backslash\{0\} .
$$

Indeed, it is enough to consider the bilateral weighted forward shift

$$
T:\left(x_{n}\right)_{n \in \mathbb{Z}} \in X \mapsto\left(w_{n-1} x_{n-1}\right)_{n \in \mathbb{Z}} \in X,
$$

where $w_{1}, w_{2}, \ldots$ are as in the previous theorem and $w_{n}:=1$ for all $n \leq 0$.

On the other hand, the next result shows that under a certain additional hypothesis DC1 implies the existence of a residual set of absolutely mean irregular vectors.

Theorem 27. If $T \in B(X)$ is distributionally chaotic of type 1 and the set $\{x \in X$ : $\left.\lim _{n \rightarrow \infty} T^{n} x=0\right\}$ is dense in $X$, then there is a residual set of absolutely mean irregular vectors for $T$.

Proof. Since $T$ is DC1, there is a residual set $R_{1}$ consisting of vectors with distributionally unbounded orbits. By Proposition 20(b),

$$
\limsup _{N \rightarrow \infty} \frac{1}{N} \sum_{j=1}^{N}\left\|T^{j} x\right\|=\infty \quad \text { for all } x \in R_{1} .
$$

On the other hand, since $\left\{x \in X: \lim _{n \rightarrow \infty} T^{n} x=0\right\}$ is dense in $X$, the set

$$
M_{k}:=\left\{x \in X: \exists N>k \text { such that } \frac{1}{N} \sum_{j=1}^{N}\left\|T^{j} x\right\|<\frac{1}{k}\right\}
$$

is open and dense in $X$, for each $k \in \mathbb{N}$. Therefore, $R_{2}:=\bigcap_{k=1}^{\infty} M_{k}$ is a residual set such that

$$
\liminf _{N \rightarrow \infty} \frac{1}{N} \sum_{j=1}^{N}\left\|T^{j} x\right\|=0 \quad \text { for all } x \in R_{2} .
$$

Thus, $R_{1} \cap R_{2}$ is a residual set consisting of absolutely mean irregular vectors for $T$.

Theorem 28. If $T \in B(X)$ is Cesàro hypercyclic and the set $\left\{x \in X: \lim _{n \rightarrow \infty} T^{n} x=0\right\}$ is dense in $X$, then there is a residual set of absolutely mean irregular vectors for $T$.

Proof. Since $T$ is Cesàro hypercyclic, there is a residual set $R_{1}$ of Cesàro hypercyclic vectors for $T$ [28, 29]. In particular, for each $x \in R_{1}$, there is a sequence $\left(N_{k}\right)$ in $\mathbb{N}$ such that

$$
\left\|\frac{1}{N_{k}} \sum_{j=1}^{N_{k}} T^{j} x\right\| \rightarrow \infty \quad \text { as } k \rightarrow \infty .
$$

Thus,

$$
\limsup _{N \rightarrow \infty} \frac{1}{N} \sum_{j=1}^{N}\left\|T^{j} x\right\|=\infty \quad \text { for all } x \in R_{1}
$$


As in the previous proof, since $\left\{x \in X: \lim _{n \rightarrow \infty} T^{n} x=0\right\}$ is dense in $X$, there is a residual set $R_{2}$ such that

$$
\liminf _{N \rightarrow \infty} \frac{1}{N} \sum_{j=1}^{N}\left\|T^{j} x\right\|=0 \quad \text { for all } x \in R_{2} .
$$

Therefore, $R_{1} \cap R_{2}$ is a residual set of absolutely mean irregular vectors for $T$.

Problem 29. Is there a Cesàro hypercyclic operator $T$ such that the set $\{x \in X$ : $\left.\lim _{n \rightarrow \infty} T^{n} x=0\right\}$ is dense in $X$ but $T$ is not DC1?

Theorem 25 also shows that the converse to Proposition 20(a) is false in general. The next result implies that the converse to Proposition 20(b) also fails.

Theorem 30. If $X=c_{0}(\mathbb{N})$ or $X=\ell^{p}(\mathbb{N})$ for some $1 \leq p \leq \infty$, then there exists an operator $T \in B(X)$ which admits an absolutely mean irregular vector whose orbit is not distributionally unbounded.

Proof. Our operator $T$ will be a unilateral weighted forward shift on $X$ with weight sequence

$$
w:=\left(B_{1} B_{2} B_{3} \ldots\right),
$$

where each block $B_{n}$ has the form

$$
B_{n}:=(\underbrace{1,1, \ldots, 1}_{2^{k_{n}}-1 \text { times }}, \underbrace{2,2, \ldots, 2}_{2 k_{n} \text { times }}, 1, \underbrace{\frac{1}{2}, \frac{1}{2}, \ldots, \frac{1}{2}}_{2 k_{n}-1 \text { times }}, \frac{n}{2(n+1)}) .
$$

The numbers $k_{n}$ will be chosen inductively. For each $n \in \mathbb{N}$, let $b_{n}:=4 k_{n}+2^{k_{n}}, s_{n}:=$ $b_{1}+\cdots+b_{n}$ and $t_{n}:=s_{n-1}+2^{k_{n}}-1$, where $s_{0}:=0$.

We start with $k_{1}:=1$, so that

$$
B_{1}=(1,2,2,1,1 / 2,1 / 4) .
$$

Suppose that $n \geq 2$ and that $k_{1}, \ldots, k_{n-1}$ have been chosen. Note that

$$
\left(\left\|T^{j} e_{1}\right\|\right)_{s_{n-1}<j \leq s_{n}}=(\underbrace{\frac{1}{n}, \frac{1}{n}, \ldots, \frac{1}{n}}_{2^{k_{n}}-1 \text { times }}, \frac{2}{n}, \frac{2^{2}}{n}, \ldots, \frac{2^{2 k_{n}}}{n}, \frac{2^{2 k_{n}}}{n}, \frac{2^{2 k_{n}-1}}{n}, \ldots, \frac{2}{n}, \frac{1}{n+1}) .
$$

As $k_{n} \rightarrow \infty$, we have that

$$
\begin{gathered}
\frac{s_{n-1}+4 k_{n}}{s_{n-1}+2^{k_{n}}-1} \rightarrow 0 \\
\frac{1}{t_{n}} \sum_{j=1}^{t_{n}}\left\|T^{j} e_{1}\right\|=\frac{\sum_{j=1}^{s_{n-1}}\left\|T^{j} e_{1}\right\|+\frac{2^{k_{n}-1}}{n}}{s_{n-1}+2^{k_{n}}-1} \rightarrow \frac{1}{n}
\end{gathered}
$$

and

$$
\frac{1}{s_{n}} \sum_{j=1}^{s_{n}}\left\|T^{j} e_{1}\right\|=\frac{\sum_{j=1}^{s_{n-1}}\left\|T^{j} e_{1}\right\|+\frac{2^{k_{n}}-1}{n}+\frac{2\left(2^{2 k_{n}+1}-2\right)}{n}+\frac{1}{n+1}}{s_{n-1}+4 k_{n}+2^{k_{n}}} \rightarrow \infty .
$$

Hence, we can choose $k_{n}$ large enough so that

$$
\frac{s_{n-1}+4 k_{n}}{t_{n}}<\frac{1}{n}
$$




$$
\frac{1}{t_{n}} \sum_{j=1}^{t_{n}}\left\|T^{j} e_{1}\right\|<\frac{2}{n}
$$

and

$$
\frac{1}{s_{n}} \sum_{j=1}^{s_{n}}\left\|T^{j} e_{1}\right\|>n
$$

By (12) and (13), $e_{1}$ is an absolutely mean irregular vector for T. By (11),

$$
\max _{t_{n}<N \leq t_{n+1}} \frac{\operatorname{card}\left(\left\{1 \leq j \leq N:\left\|T^{j} e_{1}\right\| \geq \frac{2}{n}\right\}\right)}{N}<\frac{s_{n-1}+4 k_{n}}{t_{n}}<\frac{1}{n} .
$$

This implies that

$$
\lim _{N \rightarrow \infty} \frac{\operatorname{card}\left(\left\{1 \leq j \leq N:\left\|T^{j} e_{1}\right\| \geq \delta\right\}\right)}{N}=0,
$$

for every $\delta>0$. In particular, the orbit of $e_{1}$ is not distributionally unbounded.

Remark 31. By arguing as in Remark 26, we obtain the following variation of the previous theorem: If $X=c_{0}(\mathbb{Z})$ or $X=\ell^{p}(\mathbb{Z})$ for some $1 \leq p \leq \infty$, then there exists an invertible operator $T \in B(X)$ which admits an absolutely mean irregular vector whose orbit is not distributionally unbounded.

We close this section with the following question.

Problem 32. Is there an operator $T$ which admits an absolutely mean irregular vector but is not DC1?

\section{Dense distributional chaos in Banach spaces}

It was proved in [14] that if $X$ is separable, $T \in B(X)$, the set $\left\{x \in X: \lim _{n \rightarrow \infty} T^{n} x=0\right\}$ is dense in $X$ and there exist $y \in X$ and $A \subset \mathbb{N}$ such that $\overline{\operatorname{dens}}(A)>0$ and $\lim _{j \in A}\left\|T^{j} y\right\|=\infty$, then $T$ has a dense distributionally irregular manifold. The following theorem improves this result and has many consequences.

Theorem 33. Suppose that $T \in B(X)$ satisfies

$$
T^{n} x \rightarrow 0 \quad \text { for all } x \in X_{0},
$$

where $X_{0}$ is a dense subset of $X$. If there exist $y \in X$ and $\delta>0$ such that

$$
\overline{\operatorname{dens}}\left(\left\{n \in \mathbb{N}:\left\|T^{n} y\right\| \geq \delta\right\}\right)>0
$$

then $T$ is distributionally chaotic of type 1 . If, in addition, $X$ is separable, then $T$ has a dense distributionally irregular manifold and, in particular, is densely distributionally chaotic.

Proof. In view of (14), it follows from [14, Proposition 9] that the set $R_{1}$ of all vectors with orbits distributionally near to 0 is residual.

By (15), the constant $C:=\overline{\operatorname{dens}}\left(\left\{n \in \mathbb{N}:\left\|T^{n} y\right\| \geq \delta\right\}\right)$ is strictly positive. Consider the set

$$
R_{2}:=\left\{x \in X: \overline{\operatorname{dens}}\left(\left\{n \in \mathbb{N}:\left\|T^{n} x\right\| \geq \delta / 2\right\}\right) \geq C\right\} .
$$


For each $k \in \mathbb{N}$, the set

$$
M_{k}:=\left\{x \in X: \exists n>k \text { with } \operatorname{card}\left(\left\{1 \leq j \leq n:\left\|T^{j} x\right\|>\delta-\frac{1}{k}\right\}\right)>n\left(C-\frac{1}{k}\right)\right\}
$$

is open (clearly) and dense (because $y+X_{0}$ is contained in it). Hence, $R_{2}$ is a residual set, since

$$
R_{2} \supset \bigcap_{k=1}^{\infty} M_{k} .
$$

If $z \in R_{1} \cap R_{2}$, then $F_{z, 0}^{*} \equiv 1$ and $F_{z, 0}(\delta / 2)<1$, that is, $(z, 0)$ is a distributionally chaotic pair of type 2 for $T$. By Theorem 2 , $T$ is distributionally chaotic of type 1 .

In the case $X$ is separable, [14, Theorem 15] guarantees that $T$ has a dense distributionally irregular manifold.

Corollary 34. Let $T \in B(X)$ be such that the set $\left\{x \in X: \lim _{n \rightarrow \infty} T^{n} x=0\right\}$ is dense in $X$. If $T$ has an eigenvalue $\lambda$ with $|\lambda| \geq 1$, then $T$ is distributionally chaotic of type 1.

Proof. Let $y$ be an eigenvector of $T$ associated to the eigenvalue $\lambda$. If $\delta:=\|y\|$, then

$$
\overline{\operatorname{dens}}\left(\left\{n \in \mathbb{N}:\left\|T^{n} y\right\| \geq \delta\right\}\right)>0 .
$$

Thus, by Theorem $33, T$ is distributionally chaotic of type 1 .

Corollary 35. Let $T \in B(X)$ be such that the set $\left\{x \in X: \lim _{n \rightarrow \infty} T^{n} x=0\right\}$ is dense in $X$. If $T$ has a nontrivial periodic point, then $T$ is distributionally chaotic of type 1.

Corollary 36. Let $T \in B(X)$ be such that the set $\left\{x \in X: \lim _{n \rightarrow \infty} T^{n} x=0\right\}$ is dense in $X$. If $T$ is Devaney chaotic, then $T$ is distributionally chaotic of type 1.

Remark 37. In Corollaries 34, 35 and 36 we can conclude that $T$ has a dense distributionally irregular manifold (hence it is densely distributionally chaotic) if $X$ is separable.

The following result improves [5, Theorem 3.7].

Corollary 38. Assume $X$ is complex and separable. Let $\left\{T_{t}\right\}_{t \geq 0}$ be a $C_{0}$-semigroup of operators on $X$ with infinitesimal generator $(A, D(A))$. If the following conditions hold:

(I) there is a dense subset $X_{0} \subset X$ with $\lim _{t \rightarrow \infty} T_{t} x=0$ for each $x \in X_{0}$, and

(II) there is some $\lambda \in \sigma_{p}(A)$ with $\Re(\lambda) \geq 0$,

then $\left\{T_{t}\right\}_{t \geq 0}$ has a dense distributionally irregular manifold.

Proof. Fix $t_{0}>0$. By (I), $\lim _{n \rightarrow \infty}\left(T_{t_{0}}\right)^{n} x=0$ for each $x \in X_{0}$. Moreover, by (II) and the point spectral mapping theorem for $C_{0}$-semigroups, $e^{t_{0} \lambda} \in \sigma_{p}\left(T_{t_{0}}\right)$ and $\left|e^{t_{0} \lambda}\right| \geq 1$. Thus, by Corollary 34 and Remark 37, the operator $T_{t_{0}}$ has a dense distributionally irregular manifold, which implies that the semigroup $\left\{T_{t}\right\}_{t \geq 0}$ has a dense distributionally irregular manifold [1, Remark 2]. 
A map $f: \mathbb{N} \rightarrow \mathbb{N}$ is said to be srictly increasing if $f(i)<f(j)$ whenever $i<j$. Given $f: \mathbb{N} \rightarrow \mathbb{N}$ and $g: \mathbb{N} \rightarrow \mathbb{N}$, we say that $f$ and $g$ are almost disjoint if the set $\{k \in \mathbb{N}: f(k)=g(k)\}$ is finite and $f(k) \neq g\left(k^{\prime}\right)$ whenever $k \neq k^{\prime}$. For each strictly increasing map $f: \mathbb{N} \rightarrow \mathbb{N}$ and each $1 \leq p<\infty, T_{f}: l^{p} \rightarrow l^{p}$ is the operator given by

$$
T_{f}\left(x_{1}, x_{2}, \cdots\right):=\left(x_{f(1)}, x_{f(2)}, \cdots\right) .
$$

As a consequence of Corollary 36, we solve the conjecture of [17].

Corollary 39. Let $f_{1}, f_{2}, \ldots, f_{t}$ be strictly increasing pairwise almost disjoint functions from $\mathbb{N}$ to $\mathbb{N}$ and let $c_{1}, c_{2}, \ldots, c_{t}$ be real numbers. If $T:=\sum_{i=1}^{t} c_{i} T_{f_{i}}$ is Devaney chaotic on $l^{p}$, then $T$ is densely distributionally chaotic.

Proof. Since the set $\left\{x \in \ell^{p}: \lim _{n \rightarrow \infty} T^{n} x=0\right\}$ is dense in $\ell^{p}$ and $\ell^{p}$ is separable, $T$ is densely distributionally chaotic.

The following result improves [14, Corollary 27] for Banach spaces.

Corollary 40. Let $X$ be a Banach sequence space in which $\left(e_{n}\right)_{n \in \mathbb{N}}$ is a basis. Let $\left(\omega_{n}\right)_{n \in \mathbb{N}}$ be a sequence of positive weights. Suppose that the unilateral weighted backward shift $B_{\omega}$ is an operator on $X$. If there exists a set $S \subset \mathbb{N}$ with $\overline{\operatorname{dens}}(S)>0$ such that

$$
\sum_{n \in S}\left(\prod_{\nu=1}^{n} \omega_{\nu}\right)^{-1} e_{n} \text { converges in } X,
$$

then $B_{\omega}$ is densely distributionally chaotic.

Proof. Let

$$
y:=\sum_{n \in S}\left(\prod_{\nu=1}^{n} \omega_{\nu}\right)^{-1} e_{n} .
$$

Then $B_{\omega}^{n-1} y=e_{1}+\cdots$ for all $n \in S$. Since the functional $x \rightarrow x_{1}$ is continuous, there exists $\varepsilon>0$ such that $\|x\|<\varepsilon$ implies $\left|x_{1}\right|<1$. Hence $\left\|B_{\omega}^{n-1} y\right\| \geq \varepsilon$ for all $n \in S$. Therefore,

$$
\overline{\operatorname{dens}}\left(\left\{n \in \mathbb{N}:\left\|B_{\omega}^{n} y\right\| \geq \varepsilon\right\}\right)>0 .
$$

Hence, by Theorem $33, B_{\omega}$ is densely distributionally chaotic.

Recall that $T \in B(X)$ is said to have a perfectly spanning set of eigenvectors associated to unimodular eigenvalues with respect to Lebesgue measure if for every subset $A$ of $\mathbb{T}$ with zero Lebesgue measure, the span of $\bigcup_{\lambda \in \mathbb{T} \backslash A} \operatorname{ker}(T-\lambda)$ is dense in $X$.

Corollary 41. [9, Corollary 18] If $T \in B(X)$ has a perfectly spanning set of eigenvectors associated to unimodular eigenvalues with respect to Lebesgue measure, then $T$ is distributionally chaotic of type 1.

Proof. By [7] there exists a dense set $X_{0} \subset X$ such that $T^{n} x \rightarrow 0$ for all $x \in X_{0}$. Since $T$ has unimodular eigenvalues, Corollary 34 implies that $T$ is distributionally chaotic of type 1. 


\section{$7 \quad$ DC3 in Banach spaces}

Proposition 42. If $T \in B(X)$ is distributionally chaotic of type 3, then the following properties hold:

(a) The spectrum of $T$ must intersect the unit circle.

(b) $\|T\|>1$.

(c) There exists $x \in X$ such that $\limsup _{N \rightarrow \infty} \frac{1}{N} \sum_{j=1}^{N}\left\|T^{j} x\right\|>0$.

(d) If $X$ is a Hilbert space, then $T$ is not hyponormal.

(e) $T$ is not an m-isometry.

(f) If $T$ is power bounded, then $T$ is not supercyclic.

Proof. (a) Suppose that the spectrum $\sigma(T)$ of $T$ does not intersect the unit cicle. By [20, Lemma 6.3], if $\sigma(T) \subset \mathbb{D}$ then $\lim _{n \rightarrow \infty} T^{n} x=0$ for every $x \in X$, and if $\sigma(T) \subset \mathbb{C} \backslash \overline{\mathbb{D}}$, then $\lim _{n \rightarrow \infty}\left\|T^{n} x\right\|=\infty$ for every nonzero $x \in X$. Otherwise, the sets $\sigma_{1}:=\sigma(T) \cap \mathbb{D}$ and $\sigma_{2}:=\sigma(T) \cap(\mathbb{C} \backslash \overline{\mathbb{D}})$ form a partition of $\sigma(T)$ into nonempty closed sets. By the Riesz decomposition theorem, there exist nontrivial $T$-invariant closed subspaces $M_{1}$ and $M_{2}$ of $X$ such that $X=M_{1} \oplus M_{2}, \sigma\left(\left.T\right|_{M_{1}}\right)=\sigma_{1}$ and $\sigma\left(\left.T\right|_{M_{2}}\right)=\sigma_{2}$. Thus all orbits of $T$ either converge to 0 or diverge to $\infty$. In any case, we see that $T$ is not DC3.

(b) Since $T$ is DC3, there exist $x \in X$ and $\delta>0$ such that $F_{T, x, 0}(\delta)<F_{T, x, 0}^{*}(\delta)$. This implies that both sets $A:=\left\{j \in \mathbb{N}:\left\|T^{j} x\right\| \geq \delta\right\}$ and $B:=\left\{j \in \mathbb{N}:\left\|T^{j} x\right\|<\delta\right\}$ are infinite. Hence, for any $j \in B$, there exists $n_{j} \in A$ such that $n_{j}>j$, and so

$$
\left\|T^{n_{j}-j}\right\| \geq \frac{\left\|T^{n_{j}-j}\left(T^{j} x\right)\right\|}{\left\|T^{j} x\right\|}=\frac{\left\|T^{n_{j}} x\right\|}{\left\|T^{j} x\right\|}>1 .
$$

Therefore, $\|T\|>1$.

(c) Since $T$ is DC3, there exist $x \in X$ and $\delta>0$ with $F_{T, x, 0}(\delta)<F_{T, x, 0}^{*}(\delta) \leq 1$. Since

$$
\frac{\operatorname{card}\left(\left\{1 \leq j \leq N:\left\|T^{j} x\right\| \geq \delta\right\}\right)}{N} \leq \frac{1}{\delta} \frac{1}{N} \sum_{j=1}^{N}\left\|T^{j} x\right\|,
$$

we obtain that $\limsup _{N \rightarrow \infty} \frac{1}{N} \sum_{j=1}^{N}\left\|T^{j} x\right\|>0$.

(d) It is well-known that any hyponormal operator $T$ satisfies that the sequence $\left(\left\|T^{n} x\right\|\right)_{n \in \mathbb{N}}$ is either increasing or eventually increasing or strictly decreasing, for any $x \in X$, which implies that $T$ cannot be DC3.

(e) As before, any $m$-isometry $T$ is so that the sequence $\left(\left\|T^{n} x\right\|\right)_{n \in \mathbb{N}}$ is eventually increasing, for every $x \in X$.

(f) If $T$ is power bounded and supercyclic, then all orbits of $T$ must converge to 0 [3, Theorem 2.2] and, therefore, $T$ is not DC3. 
Corollary 43. Let $T \in B(X)$. If

$$
\lim _{N \rightarrow \infty} \frac{1}{N} \sum_{j=1}^{N}\left\|T^{j} x\right\|=0 \quad \text { for any } x \in X,
$$

then $T$ is not distributionally chaotic of type 3.

Let us now give an example of a power bounded DC3 operator on the Hilbert space. Note that a power bounded operator cannot be DC2 $\frac{1}{2}$, since every $\mathrm{DC} 2 \frac{1}{2}$ operator is Li-Yorke chaotic.

Example 44. There exists a DC3 operator $T$ on the Hilbert space $\ell^{2}(\mathbb{N})$ which is power bounded. In particular, $T$ is not Li-Yorke chaotic.

Let $\left(e_{i}\right)_{i \in \mathbb{N}}$ be the canonical basis of $\ell^{2}(\mathbb{N})$. We select a fast increasing sequence $\left(s_{n}\right)_{n \in \mathbb{N}}$ of positive integers so that $s_{1}=1$ and $\lim _{n \rightarrow \infty} s_{n} / s_{n+1}=0$, we fix a constant $\alpha>1$, and we define $T: \ell^{2}(\mathbb{N}) \rightarrow \ell^{2}(\mathbb{N})$ by

$$
T e_{i}= \begin{cases}e_{i+1}, & \text { if } \left.i \in \cup_{n \in \mathbb{N}}\right] s_{n}, s_{n+1}[, \\ \frac{1}{\alpha} e_{i+1}, & \text { if } i \in\left\{s_{2 n-1}: n \in \mathbb{N}\right\}, \\ \alpha e_{i+1}, & \text { if } i \in\left\{s_{2 n}: n \in \mathbb{N}\right\} .\end{cases}
$$

It is not difficult to check that the following statements hold:

(a) $T$ is a continuous linear operator on $\ell^{2}(\mathbb{N})$;

(b) $\left\|T^{n}\right\|=\alpha$ for all $n \in \mathbb{N}$;

(c) $T^{i} e_{1}=\frac{1}{\alpha} e_{i+1}$ for $i \in \bigcup_{n \in \mathbb{N}}\left[s_{2 n-1}, s_{2 n}[\right.$;

(d) $T^{i} e_{1}=e_{i+1}$ for $i \in \bigcup_{n \in \mathbb{N}}\left[s_{2 n}, s_{2 n+1}[\right.$.

It follows that $T$ is power bounded. In particular, $T$ is not Li-Yorke chaotic. For any $\delta \in] 1 / \alpha, 1[$, property (c) implies that

$$
\begin{aligned}
F_{T, e_{1}, 0}^{*}(\delta) & \geq \limsup _{n \rightarrow \infty} \frac{1}{s_{2 n}} \operatorname{card}\left(\left\{1 \leq i \leq s_{2 n}:\left\|T^{i} e_{1}\right\|<\delta\right\}\right) \\
& \geq \limsup _{n \rightarrow \infty} \frac{s_{2 n}-s_{2 n-1}}{s_{2 n}}=1,
\end{aligned}
$$

and property (d) implies that

$$
\begin{aligned}
F_{T, e_{1}, 0}(\delta) & \leq \liminf _{n \rightarrow \infty} \frac{1}{s_{2 n+1}} \operatorname{card}\left(\left\{1 \leq i \leq s_{2 n+1}:\left\|T^{i} e_{1}\right\|<\delta\right\}\right) \\
& \leq \liminf _{n \rightarrow \infty} \frac{s_{2 n}}{s_{2 n+1}}=0 .
\end{aligned}
$$

Thus, $T$ is distributionally chaotic of type 3 .

As we have just seen, in general, DC3 does not imply Li-Yorke chaos. The next theorem, which complements Theorem 8, shows that under a certain additional condition DC3 actually implies DC1. 
Theorem 45. If $T \in B(X)$ and there exists a dense set $X_{0} \subset X$ such that

$$
T^{n} x \rightarrow 0 \quad \text { for all } x \in X_{0},
$$

then properties (i)-(vi) in Theorem 8 are also equivalent to:

(vii) $T$ is distributionally chaotic of type 3 ;

(viii) $T$ admits a distributionally chaotic pair of type 3 .

Proof. It is enough to prove that (viii) $\Rightarrow$ (iii). By (viii), there exist $y \in X$ and $\delta>0$ such that $F_{T, y, 0}(\delta)<1$, which is equivalent to $\overline{\operatorname{dens}}\left(\left\{n \in \mathbb{N}:\left\|T^{n} y\right\| \geq \delta\right\}\right)>0$. Thus, by Theorem 33, $T$ is distributionally chaotic of type 1 .

Corollary 46. Let $X$ be a Banach sequence space in which $\left(e_{n}\right)$ is a basis ([22], Section 4.1). Suppose that the unilateral weighted backward shift

$$
B_{w}\left(x_{1}, x_{2}, x_{3}, \ldots\right):=\left(w_{2} x_{2}, w_{3} x_{3}, w_{4} x_{4}, \ldots\right)
$$

is an operator on $X$. Then $B_{w}$ is DC3 if and only if $B_{w}$ is DC1.

Proof. Since the orbit of any sequence with finite support converges to zero, we can apply the above theorem.

Remark 47. Note that the operator constructed in Example 44 is a unilateral weighted forward shift on $\ell^{2}(\mathbb{N})$. Thus, such a shift can be DC3 and not DC $2 \frac{1}{2}$, contrary to what happens in the case of unilateral weighted backward shifts (Corollary 46).

Example 48. There exist mixing operators which are not DC3.

(A) Let $T \in B\left(\ell^{1}(\mathbb{N})\right)$ be one of the operators considered in Example $23((\mathrm{~A})$ or $(\mathrm{B}))$. We know that $T$ is mixing and absolutely Cesàro bounded. By Corollary 21, $T$ is not DC $2 \frac{1}{2}$. Since the set $\left\{x \in \ell^{1}(\mathbb{N}): \lim _{n \rightarrow \infty} T^{n} x=0\right\}$ is dense in $\ell^{1}(\mathbb{N})$, we conclude from Theorem 45 that $T$ is not DC3.

(B) The example from [33] mentioned in Example 23 (C) is mixing and, since for each $x \in \ell^{p}(v)$ and each $\varepsilon>0$,

$$
\lim _{n \rightarrow \infty} \frac{\operatorname{card}\left(\left\{1 \leq j \leq n:\left\|B^{j} x\right\|<\varepsilon\right\}\right)}{n}=1,
$$

we have that $B$ is not DC3.

Example 49. There exist invertible DC1 operators $T$ such that $T^{-1}$ is not DC3.

(A) Let $T$ be the bilateral weighted forward shift on $\ell^{1}(\mathbb{Z})$ with weights

$$
w_{k}:=2 \text { for } k<1, \quad w_{k}:=\frac{2 k-1}{2 k} \text { for } k \geq 1 .
$$

Since $\lim _{n \rightarrow \infty} \prod_{i=1}^{n} w_{i}=0$ and $\sum_{n \in \mathbb{N}}\left(\prod_{i=-n+1}^{0} w_{i}\right)^{-1} e_{-n}$ converges in $\ell^{1}(\mathbb{Z})$, it follows from [14, Corollary 31] that $T$ is DC1. Now, $T^{-1}$ is the bilateral weighted backward shift on $\ell^{1}(\mathbb{Z})$ with weights

$$
\lambda_{n}:=\frac{1}{w_{n-1}} \quad(n \in \mathbb{Z}),
$$

and this operator is absolutely Cesàro bounded [25]. Since the set $\left\{x \in \ell^{1}(\mathbb{Z})\right.$ : $\left.\lim _{n \rightarrow \infty} T^{-n} x=0\right\}$ is dense in $\ell^{1}(\mathbb{Z}), T^{-1}$ is not DC3. 
(B) In [26, Corollary 7] it was shown that there exists an invertible operator $T$ on $\ell^{2}(\mathbb{N})$ of the form $T=I+K$, with $K$ compact, such that $T$ is DC1, but $T^{-1}$ is not DC1. The proof actually yields that $T^{-1}$ is not DC3.

We leave two more open problems. The first one is a stronger version of Problem 17.

Problem 50. Is there a Devaney chaotic operator on a Banach space which is not DC3?

The second one might seem surprising, because any notion of chaos is not compatible with linearity in finite dimensional spaces.

Problem 51. Is there a DC3 operator on a finite dimensional space?

Remark 52. Concerning the last problem, it is not difficult to show that any finite dimensional operator whose matrix on an orthonormal basis has the Jordan form cannot be DC3. It is true that every matrix is similar to a Jordan model, but we also don't know whether DC3 is invariant under similarities. We should mention that, even in the case that we have an invariant subspace for which all the orbits are bounded, this fact does not avoid in principle the possibility that DC3 is satisfied.

We conclude with a diagram that contains the implications that we know, under the assumption that there is a dense subset of vectors whose orbits converge to 0 .

Remark: If $\left\{x: T^{n} x \rightarrow 0\right\}$ is a dense set of $X$, then:

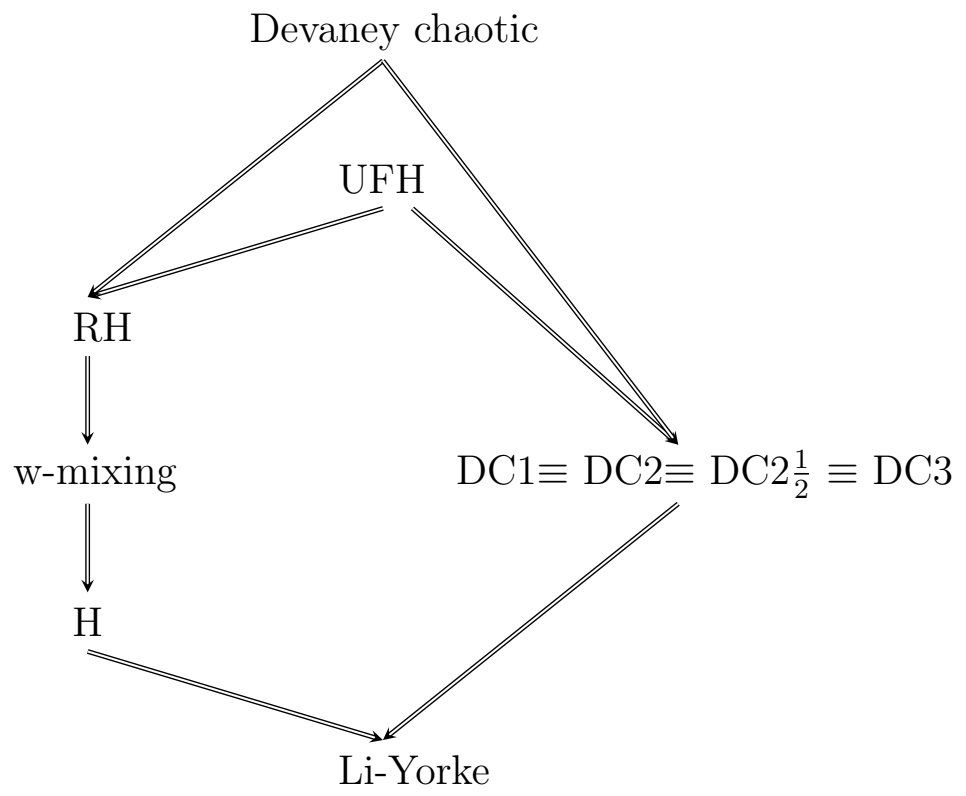

Figure 2: Implications between different definitions related with hypercyclicity and caos in Banach spaces when $\left\{x: T^{n} x \rightarrow 0\right\}$ is a dense set of $X$.

\section{Acknowledgements}

This work was partially done on a visit of the first author to the Departament de Matemàtica Aplicada at Universitat Politècnica de València (Spain), and he is very grateful for 
the hospitality. The first author was supported by CNPq (Brazil) and by the EBW+ Project (Erasmus Mundus Programme). The second and the third authors were supported by MINECO, Projects MTM2013-47093-P and MTM2016-75963-P, and by GVA, Project PROMETEOII/2013/013. The fourth author was supported by the scientific research starting project of Southwest Petroleum University (No. 2015QHZ029) and the National Natural Science Foundation of China (No. 11601449).

\section{References}

[1] A. A. Albanese, X. Barrachina, E. M. Mangino and A. Peris, Distributional chaos for strongly continuous semigroups of operators, Commun. Pure Appl. Anal. 12 (2013), no. 5, 2069-2082.

[2] A. Aleman and L. Suciu, On ergodic operator means in Banach spaces, Integral Equations Operator Theory 85 (2016), no. 2, 259-287.

[3] S. I. Ansari and P. S. Bourdon, Some properties of cyclic operators, Acta Sci. Math. (Szeged) 63 (1997), no. 1-2, 195-207.

[4] F. Balibrea, J. Smítal and M. Štefánková, The three versions of distributional chaos, Chaos Solitons Fractals 23 (2005), no. 5, 1581-1583.

[5] X. Barrachina and J. A. Conejero, Devaney chaos and distributional chaos in the solution of certain partial differential equations, Abstr. Appl. Anal. 2012, Art. ID 457019, 11pp.

[6] F. Bayart and S. Grivaux, Frequently hypercyclic operators, Trans. Amer. Math. Soc. 358 (2006), no. 11, 5083-5117.

[7] F. Bayart and S. Grivaux, Hypercyclicity and unimodular point spectrum, J. Funct. Anal. 226 (2005), no. 2, 281-300.

[8] F. Bayart and É. Matheron, Dynamics of Linear Operators, Cambridge University Press, Cambridge, 2009.

[9] F. Bayart and I. Z. Ruzsa, Difference sets and frequently hypercyclic weighted shifts, Ergodic Theory Dynam. Systems 35 (2015), no. 3, 691-709.

[10] M. J. Beltrán, Operators on weighted spaces of holomorphic functions, PhD Thesis, Universitat Politècnica de València, 2014.

[11] M. J. Beltrán, J. Bonet, C. Fernández, Classical operators on weighted Banach spaces of entire functions, Proc. Amer. Math. Soc. 141 (2013), 4293-4303.

[12] M. J. Beltrán, M. C. Gómez-Collado, E. Jordá, D. Jornet, Mean ergodic composition operators on Banach spaces of holomorphic functions, J. Funct. Anal. 270 (2016), $4369-4385$.

[13] T. Bermúdez, A. Bonilla, F. Martínez-Giménez and A. Peris, Li-Yorke and distributionally chaotic operators, J. Math. Anal. Appl. 373 (2011), no. 1, 83-93. 
[14] N. C. Bernardes Jr., A. Bonilla, V. Müller and A. Peris, Distributional chaos for linear operators, J. Funct. Anal. 265 (2013), no. 9, 2143-2163.

[15] N. C. Bernardes Jr., A. Bonilla, V. Müller and A. Peris, Li-Yorke chaos in linear dynamics, Ergodic Theory Dynam. Systems 35 (2015), no. 6, 1723-1745.

[16] J. Bès, Q. Menet, A. Peris and Y. Puig, Recurrence properties of hypercyclic operators, Math. Ann. 366 (2016), no. 1-2, 545-572.

[17] D. Bongiorno, U. B. Darji and L. Di Piazza, Rolewicz-type chaotic operators, J. Math. Anal. Appl. 431 (2015), no. 1, 518-528.

[18] A. Bonilla and K.-G. Grosse-Erdmann, Frequently hypercyclic operators and vectors, Ergodic Theory Dynam. Systems 27 (2007), no. 2, 383-404. Erratum: Ergodic Theory Dynam. Systems 29 (2009), no. 6, 1993-1994.

[19] K.-J. Engel and R. Nagel, One-Parameter Semigroups for Linear Evolution Equations, Springer-Verlag, New York - Berlin, 2000.

[20] N. S. Feldman, V. G. Miller and T. L. Miller, Hypercyclic and supercyclic cohyponormal operators, Acta Sci. Math. (Szeged) 68 (2002), no. 3-4, 965-990.

[21] S. Grivaux and É. Matheron, Invariant measures for frequently hypercyclic operators, Adv. Math. 265 (2014), 371-427.

[22] K.-G. Grosse-Erdmann and A. Peris Manguillot, Linear Chaos, Springer, London, 2011.

[23] J. Hantáková, S. Roth and Z. Roth, On the weakest version of distributional chaos, Int. J. Bifurcation Chaos 26 (2016), 1650235.

[24] B. Hou, P. Cui and Y. Cao, Chaos for Cowen-Douglas operators, Proc. Amer. Math. Soc. 138 (2010), no. 3, 929-936.

[25] B. Hou and L. Luo, Some remarks on distributional chaos for bounded linear operators, Turkish J. Math. 39 (2015), no. 2, 251-258.

[26] B. Hou and L. Luo, $C^{*}$ algebra and inverse chaos, ArXiv:1503.06750v3.

[27] W. Huang, J. Li and X. Ye, Stable sets and mean Li-Yorke chaos in positive entropy systems, J. Funct. Anal. 266 (2014), no. 6, 3377-3394.

[28] F. León-Saavedra, Operators with hypercyclic Cesàro means, Studia Math. 152 (2002), no. 3, 201-215.

[29] F. León-Saavedra and V. Müller, Hypercyclic sequences of operators, Studia Math. 175 (2006), no. 1, 1-18.

[30] J. Li, S. Tu and X. Ye, Mean equicontinuity and mean sensitivity, Ergodic Theory Dynam. Systems 35 (2015), no. 8, 2587-2612.

[31] T. Y. Li and J. A. Yorke, Period three implies chaos, Amer. Math. Monthly 82 (1975), no. 10, 985-992. 
[32] F. Martínez-Giménez, P. Oprocha and A. Peris, Distributional chaos for backward shifts, J. Math. Anal. Appl. 351 (2009), no. 2, 607-615.

[33] F. Martínez-Giménez, P. Oprocha and A. Peris, Distributional chaos for operators with full scrambled sets, Math. Z. 274 (2013), no. 1-2, 603-612.

[34] Q. Menet, Linear chaos and frequent hypercyclicity, Trans. Amer. Math. Soc. 369 (2017), 4977-4994.

[35] P. Oprocha, Distributional chaos revisited, Trans. Amer. Math. Soc. 361 (2009), no. 9, 4901-4925.

[36] B. Schweizer and J. Smítal, Measures of chaos and a spectral decomposition of dynamical systems on the interval, Trans. Amer. Math. Soc. 344 (1994), no. 2, $737-754$.

[37] J. Smítal and M. Štefánková, Distributional chaos for triangular maps, Chaos Solitons Fractals 21 (2004), no. 5, 1125-1128.

[38] X. Wu, G. Chen and P. Zhu, Invariance of chaos from backward shift on the Köthe sequence space, Nonlinearity 27 (2014), 271-288.

[39] X. Wu, P. Oprocha and G. Chen, On various definitions of shadowing with average error in tracing, Nonlinearity 29 (2016), no. 7, 1942-1972.

[40] X. Wu, L. Wang and G. Chen, Weighted backward shift operators with invariant distributionally scrambled subsets, Annals of Functional Analysis (accepted for publication).

[41] X. Wu and P. Zhu, On the equivalence of four chaotic operators, Appl. Math. Lett. 25 (2012), no. 3, 545-549.

[42] X. Wu and P. Zhu, Dense chaos and densely chaotic operators, Tsukuba J. Math. 36 (2012), no. 2, 367-375.

N. C. Bernardes Jr.

Departamento de Matemática Aplicada, Instituto de Matemática, Universidade Federal do Rio de Janeiro, Caixa Postal 68530, Rio de Janeiro, RJ 21945-970, Brazil.

e-mail: ncbernardesjr@gmail.com

A. Bonilla

Departamento de Análisis Matemático, Universidad de la Laguna, 38271, La Laguna (Tenerife), Spain.

e-mail: abonilla@ull.es

A. Peris

Institut Universitari de Matemàtica Pura i Aplicada, Universitat Politècnica de València, Edifici 8E, Acces F, 4a planta, 46022 València, Spain.

e-mail: aperis@mat.upv.es

$\mathrm{X} . \mathrm{Wu}$

School of Sciences and Research Center for Mathematics and Mechanics, Southwest Petroleum University, Chengdu, Sichuan, 610500, People's Republic of China.

e-mail: wuxinxing5201314@163.com 